\title{
HAMILTON JACOBI EQUATIONS WITH OBSTACLES
}

\author{
ROGER ROBYR AND CAMILLO DE LELLIS
}

\begin{abstract}
We consider a problem in the theory of optimal control proposed for the first time by Bressan. We characterize the associated minimum time function using tools from geometric measure theory and we obtain as a corollary an existence theorem for a related variational problem.
\end{abstract}

\section{INTRODUCTION}

In this paper we deal with a problem in the theory of optimal control introduced for the first time by Alberto Bressan in [5] and which has been subsequently studied in several papers (see [6], [7], [8] and [9]). The problem models the spread of fire in a forest or that of a contaminating agent.

Consider a continuous multifunction $F: \mathbb{R}^{2} \mapsto \mathbb{R}^{2}$ with compact, convex values (that is, $F(x)$ is a compact convex set for every $x$ and $F\left(x_{n}\right) \rightarrow F(x)$ in the sense of Hausdorff when $\left.x_{n} \rightarrow x\right)$. A bounded, open set $R_{0} \subset \mathbb{R}^{2}$ is the initial contaminated set and $F$ describes the speed at which the contamination might spread. A controller can construct one-dimensional rectifiable sets $\gamma$ (or "walls") which block the spreading of the contamination, without exceeding a certain length. More precisely, consider a continuous function $\psi: \mathbb{R}^{2} \mapsto \mathbb{R}_{+}$ and a constant $\psi_{0}$ with $\psi \geq \psi_{0}>0$. We denote by $\gamma(t) \subset \mathbb{R}^{2}$ the portion of the wall constructed within time $t \geq 0$ and we make the following assumptions $\left(\mathcal{H}^{1}\right.$ denotes the one-dimensional Hausdorff measure):

(H1) $\gamma\left(t_{1}\right) \subseteq \gamma\left(t_{2}\right)$ for every $0 \leq t_{1} \leq t_{2}$;

(H2) $\int_{\gamma(t)} \psi d \mathcal{H}^{1} \leq t$ for every $t \geq 0$.

A strategy $\gamma$ satisfying (H1)-(H2) will be called an admissible strategy.

At each time $t$, the contaminated set consists of the points reached by absolutely continuous trajectories $x(\cdot)$ which start in $R_{0}$, solve the differential inclusion $\dot{x} \in F(x)$ and do not cross the walls $\gamma$. That is,

$$
\begin{aligned}
& R^{\gamma}(t):=\left\{x(t) \mid x \in W^{1,1} \cap C\left([0, t], \mathbb{R}^{2}\right), x(\tau) \notin \gamma(\tau) \quad \forall \tau,\right. \\
& \left.x(0) \in R_{0} \text { and } \dot{x}(\tau) \in F(x(\tau)) \text { for a.e. } \tau\right\} .
\end{aligned}
$$

The purpose of this paper is to study the minimum time function at which a point gets contaminated. We will be able to characterize this function via a suitable modification of the usual Hamilton-Jacobi partial differential equation. In the paper [7] Bressan and the first author introduced a variational problem on the set of admissible strategies and proved the existence of a minimizer (this problem is connected to that of confining the 
fire in a bounded set, see for instance [8]). An interesting byproduct of our analysis is a shorter proof of this existence result. The prize to pay is the use of some more advanced techniques in geometric measure theory.

1.1. Minimum time function. Given an admissible strategy $\gamma$, for any $x \in \mathbb{R}^{2}$ we set

$$
T^{\gamma}(x):=\inf \left\{t>0: x \in R^{\gamma}(t)\right\} .
$$

$T^{\gamma}(x)$ is the time at which the contaminating agent reaches $x$. Obviously $T^{\gamma}$ vanishes identically on $R_{0}$ and the total contaminated set is given by $\left\{T^{\gamma}<+\infty\right\}$.

If $\gamma(t)=\emptyset$ for every $t$, then $T^{\gamma}$ is the minimum time function of a classical control problem. Let us introduce the hamiltonian function related to it.

Definition 1.1. $H(x, p):=\sup _{q \in F(x)}\{p \cdot q\}-1$.

In what follows, we will always assume that

(H3) There is a constant $\lambda>0$ s.t. $B_{\lambda}(0) \subset F(x)$ for all $x$.

It is well known that, under (H3) and the assumption $\gamma=\emptyset, T^{\gamma}$ is a Lipschitz map and satisfies the Hamilton-Jacobi equation

$$
H\left(x, \nabla T^{\gamma}(x)\right)=0 \quad \text { for a.e. } x \in \mathbb{R}^{2} \backslash R_{0} .
$$

Indeed, $T^{\gamma}$ is characterized as the viscosity solution of (3) in $\mathbb{R}^{2} \backslash \bar{R}_{0}$ with boundary value equal to 0 (see for instance [11] or [4]).

Assume for the moment that $\gamma_{\infty}:=\cup_{t} \gamma(t)$ is a sufficiently regular curve. Then $T^{\gamma}$ must be a viscosity solution of (3) in $\left\{T^{\gamma}<\infty\right\} \backslash\left(\bar{R}_{0} \cup \gamma_{\infty}\right)$. Moreover, $T^{\gamma}$ has jump discontinuities on $\gamma_{\infty}$. We can regard it as a "viscosity solution of (3) with obstacles $\gamma_{\infty}$ ". In this note we propose a suitable mathematical definition of this concept and use it to characterize $T^{\gamma}$. The strength of our result is its generality, which will give us a few interesting corollaries. In order to state our main theorem, we need some notation.

1.2. Main Theorem. We start by introducing the "complete strategies", which were first defined in [7]. The definition is motivated by the following example. Assume that $\gamma$ is an admissible strategy and consider a family of sets $\eta(t)$ satisfying (H1) and $\mathcal{H}^{1}(\eta(t))=0$ for every $t$. Then $\gamma(t) \cup \eta(t)$ satisfies (H1)-(H2). In other words, given an admissible strategy $\gamma$, we can increase its effectiveness by adding an $\mathcal{H}^{1}$-negligible amount of walls.

Definition 1.2. An admissible strategy $\gamma$ is complete if

(i) $\gamma(t)=\bigcap_{s>t} \gamma(s)$;

(ii) $\gamma(t)$ contains all its points of positive upper density, i.e. all $x$ s.t.

$$
\limsup _{r \downarrow 0} \frac{\mathcal{H}^{1}\left(B_{r}(x) \cap \gamma(t)\right)}{r}>0 .
$$

The following proposition follows from standard geometric measure theory.

Proposition 1.3 (Lemma 4.2 of [7]). Let $\gamma$ be an admissible strategy. Then there exists a complete admissible strategy $\gamma^{c}$ such that 
(iii) $\gamma(t) \subset \gamma^{c}(t)$;

(iv) $\mathcal{H}^{1}\left(\gamma^{c}(t) \backslash \gamma(t)\right)=0$ except for a countable number of times $t$.

An interesting byproduct of the results of this note is a proof of the intuitive fact that $\gamma^{c}$ has the maximum effectiveness among all strategies which differ from $\gamma$ by a negligible amount of walls (that is, $\gamma^{c}$ has the largest minimum time function in this set of strategies, cp. with Theorem 1.5 below).

We next introduce some notation in order to describe our "viscosity solution" to the Hamilton-Jacobi equation with obstacles.

Definition 1.4. Given a measurable function $u: \mathbb{R}^{2} \rightarrow[0, \infty]$ and a $t \in[0, \infty[$ we set $u_{t}:=u \wedge t=\min \{u, t\}$.

For a given strategy $\gamma$, a measurable $u: \mathbb{R}^{2} \rightarrow[0, \infty]$ belongs to the class $\mathcal{S}^{\gamma}$ if the following conditions hold for every $t \in[0, \infty[$ :

(a) $u_{t} \in S B V_{l o c}\left(\mathbb{R}^{2}\right), \mathcal{H}^{1}\left(J_{u_{t}} \backslash \gamma(t)\right)=0$ and $u_{t} \equiv 0$ on $R_{0}$;

(b) If $\nabla u_{t}$ denotes the absolutely continuous part of $D u_{t}$, then

$$
H\left(x, \nabla u_{t}(x)\right) \leq 0 \quad \text { for a.e. } x .
$$

$S B V_{\text {loc }}\left(\mathbb{R}^{2}\right)$ is a linear subspace of $B V_{l o c}\left(\mathbb{R}^{2}\right)$ (where the latter is the space of functions having bounded variation on every bounded open subset of $\mathbb{R}^{2}$ ). For its precise definition we refer to the next Section. We are now ready to state the main result of this paper.

Theorem 1.5. Let $\gamma$ be an admissible strategy. Assume (H1), (H2), (H3) and

(H4) the initial set $R_{0}$ is open and $\partial R_{0}$ has zero 2-dimensional Lebesgue measure. Then $T^{\gamma} \in \mathcal{S}^{\gamma}$ and $T^{\gamma^{c}}$ is the unique maximal element of $\mathcal{S}^{\gamma}$, that is

$$
\text { for every } v \in \mathcal{S}^{\gamma} \text { we have } v \leq T^{\gamma^{c}} \text { a.e.. }
$$

1.3. A variational problem. Besides its intrinsic interest, Theorem 1.5, together with the SBV compactness theorem of Ambrosio and De Giorgi, yields a direct proof of the existence of minima for the variational problem first studied in [7]. More precisely, consider two continuous, non-negative functions $\alpha, \beta: \mathbb{R}^{2} \mapsto \mathbb{R}_{+}$and define

$$
\begin{array}{rrr}
R_{\infty}^{\gamma}:=\bigcup_{t>0} R^{\gamma}(t), & \gamma_{\infty}:=\bigcup_{t>0} \gamma(t) & \text { and } \\
J(\gamma) & :=\int_{R_{\infty}^{\gamma}} \alpha d \mathcal{L}^{2}+\int_{\gamma_{\infty}} \beta d \mathcal{H}^{1}, &
\end{array}
$$

Note that the functional $J$ is well defined: the set $R_{\infty}^{\gamma}$ is indeed measurable by Theorem 1.5 because $R_{\infty}^{\gamma}=\left\{T^{\gamma}<\infty\right\}$ (however, the measurability of $R_{\infty}^{\gamma}$ can also be proved directly; cp. with Lemma 3.1 of [7]). As a consequence of Theorem 1.5 we have the following.

Corollary 1.6 (Cp. with Theorem 1.1 of [7]). In addition to (H1)-(H4) assume that:

(H5) $\alpha \geq 0, \beta \geq 0, \alpha$ is locally integrable and $\beta$ is lower semicontinuous.

Then, there exists a strategy that minimizes $J$ among all the admissible ones. 


\section{Preliminaries on BV functions}

Most of this section will be devoted to prove the following technical proposition, which is a key point of our proof. We refer below for the definition of approximate continuity.

Proposition 2.1. Let $u \in \mathcal{S}^{\gamma}$ and assume $\gamma$ is a complete strategy. Then there is a measurable function $\tilde{u}$ having the following properties:

(i) $u=\tilde{u}$ a.e. (i.e. $\tilde{u}$ is a representative of $u$ );

(ii) $\tilde{u}_{t}$ is approximately continuous at every $x \notin \gamma(t)$;

(iii) If $\Phi:[0,1] \times[0,1] \rightarrow \mathbb{R}^{2}$ is a $C^{1}$ diffeomorphism (of $[0,1]^{2}$ with its image) and $\alpha_{\tau}$ denotes the curve $\{\Phi(\tau, s): s \in[0,1]\}$, then the following holds for a.e. $\tau$ and for every $t$ :

$$
\left.\begin{array}{c}
\text { If } \alpha_{\tau} \cap \gamma(t)=\emptyset \text {, then } w(\cdot):=\tilde{u}_{t}(\Phi(\tau, \cdot)) \text { is Lipschitz and } \\
\dot{w}(s)=\nabla u_{t}(\Phi(\tau, s)) \cdot \partial_{s} \Phi(\tau, s) \quad \text { for a.e. } s \\
H\left(\Phi(\tau, s), \nabla u_{t}(\Phi(\tau, s))\right) \leq 0 \quad \text { for a.e. } s .
\end{array}\right\}
$$

In the proposition above it is crucial that the Lipschitz regularity holds for $w$ in its pointwise definition: we do not need to redefine it on a set of measure zero!

A second technical point is the next proposition. This time, however, the statement is a well-known fact for BV functions and we refer to the monograph [1]. In what follows, the derivative of BV functions $v$, which are Radon measures, will be decomposed into its absolutely continuous part and its singular part, using the notation $D v=\nabla v \mathcal{L}^{2}+D^{s} v$.

Theorem 2.2 (Approximate Differentiability). Let $v$ be a $B V(\Omega)$ function and $D v=$ $\nabla v \mathcal{L}^{n}+D^{s} v$. Then, at a.e. $x \in \Omega$ there exists a measurable set $B$ (possibly depending on x) such that:

$$
\begin{aligned}
& \text { (i) } \quad \lim _{r \downarrow 0} \frac{\mathcal{L}^{n}\left(B_{r}(x) \backslash B\right)}{r^{n}}=0 ; \\
& \text { (ii) } \quad \lim _{z \rightarrow x, z \in B} \frac{v(z)-v(x)-\langle\nabla v(x),(z-x)\rangle}{|z-x|}=0 .
\end{aligned}
$$

Or, in the language of [12], $v$ is approximately differentiable at a.e. $x$ with approximate differential given by $\nabla v(x)$.

2.1. Decomposition of $D u$, SBV functions and slicing. We list here several fine properties of BV functions which will play a crucial role throughout the paper. From now on, given a Radon measure $\mu$ on a Borel set $E \subset \mathbb{R}^{n}$, we will denote its total variation on $E$ by $\|\mu\|_{T V(E)}$. If $u$ is a $\mathrm{BV}$ function, the singular part of $D u$, namely the measure $D^{s} u$, can be further decomposed into, respectively, a Cantor part and a jump part, i.e. $D^{s} u=D^{c} u+f \nu \mathcal{H}^{n-1}\left\llcorner J_{u}\right.$, where:

- $J_{u}$ is the jump set of $u$ and it is a rectifiable set of dimension $n-1$;

- $\mathcal{H}^{n-1}\left\llcorner J_{u}\right.$ denotes the measure $\mu$ s.t. $\mu(E)=\mathcal{H}^{n-1}\left(J_{u} \cap E\right)$;

- $\nu$ is a Borel vector field orthogonal to $J_{u}$ and with $|\nu|=1$; 
- $f$ is a Borel scalar function;

- $D^{c} u(E)=0$ for every Borel set $E$ with $\mathcal{H}^{n-1}(E)<\infty$.

A BV function $u$ belongs to SBV if $D^{c} u$ vanishes. We refer to Chapter 3 of [1] for the details.

In the case of one-dimensional BV functions, the jump set $J_{u}$ consists of countably many points. The measure $D u$ will then be denoted by $\frac{d u}{d s}$ and we will use $u^{\prime}$ for the $L^{1}$ function $\nabla u$. The decomposition above reads then as

$$
\frac{d u}{d s}=u^{\prime} \mathcal{L}^{1}+\sum_{s_{i} \in J_{u}} f\left(s_{i}\right) \delta_{s_{i}}+D^{c} u .
$$

Each $f\left(s_{i}\right)$ is, thus, a real number and $D^{c} u$ is the singular nonatomic part of the measure $\frac{d u}{d s}$ (see Section 3.2 of [1]).

Next, recall the following theorem (cp. with Section 3.11 of [1]).

Theorem 2.3 (Slicing). A function $u \in L^{1}\left([0,1]^{2}\right)$ belongs to $B V$ iff

(1) The functions $u(y, \cdot)$ and $u(\cdot, y)$ belong to $B V([0,1])$ for a.e. $y$;

(2) The following integral is finite

$$
\int\left(\left\|\frac{d}{d s} u(y, \cdot)\right\|_{T V([0,1])}+\left\|\frac{d}{d s} u(\cdot, y)\right\|_{T V([0,1])}\right) d y .
$$

The function $u$ belongs to $S B V$ if and only if the two conditions above hold and, in addition

(3) $u(y, \cdot)$ and $u(\cdot, y)$ belong to $S B V$ for a.e. $y$.

Moreover, if $u \in S B V$ and we write $D u=\nabla u \mathcal{L}^{2}+f \nu \mathcal{H}^{1}\left\llcorner J_{u}\right.$, the following identity is valid for a.e. $y \in[0,1]$ :

$$
\frac{d}{d s} u(y, \cdot)=\langle\nabla u,(0,1)\rangle \mathcal{L}^{1}+\sum_{s_{i} \in J(y)} \alpha_{i} \delta_{s_{i}},
$$

where $J(y):=\left\{s:(y, s) \in J_{u}\right\}$ and $\alpha_{i}=f\left(y, s_{i}\right)\left\langle\nu\left(y, s_{i}\right),(0,1)\right\rangle$.

Remark 2.4. The obvious modification of Theorem 2.3 holds in coordinates which are locally $C^{1}$-diffeomorphic to the cartesian ones. For instance the theorem holds in polar coordinates (except at the origin).

2.2. Fine properties of $1-\mathbf{d} B V$ functions. When $I$ is an interval and $u \in B V(I)$, we can change the values of $u$ on a set of zero Lebesgue measure so to gain a function $\tilde{u}$ with the following properties (see Section 3.2 of [1]):

- $\tilde{u}$ is continuous at every point $t \in I \backslash J_{u}$;

- $u^{+}(t)=\lim _{\tau \downarrow t} \tilde{u}(\tau)$ and $u^{-}(t)=\lim _{\tau \uparrow t} \tilde{u}(\tau)$ exist (and are finite) at every $t \in J_{u}$.

Moreover, the coefficients $f\left(s_{i}\right)$ of (10) satisfy $f\left(s_{i}\right)=u^{+}\left(s_{i}\right)-u^{-}\left(s_{i}\right)$. It is customary to set $\tilde{u}\left(s_{i}\right):=\left(u^{+}\left(s_{i}\right)+u^{-}\left(s_{i}\right)\right) / 2$. $\tilde{u}$ is then called the precise representative of $u$. The following Proposition is a simple corollary of the properties of the precise representative. 
Proposition 2.5. If $I$ is an interval, $u \in B V(I)$ and $J_{u}=\emptyset$, then the precise representative $\tilde{u}$ is continuous. If in addition $u \in S B V(I)$, then $\tilde{u} \in W^{1,1} \cap C$ and its distributional derivative is the $L^{1}$ function $u^{\prime}$.

2.3. More on fine properties. The properties listed above for 1-d $B V$ functions can be suitably generalized to the higher-dimensional case. In order to do that we must introduce the concept of approximate continuity.

Definition 2.6. A measurable map $u: \mathbb{R}^{n} \supset E \rightarrow[-\infty,+\infty]$ is said approximately continuous at $x \in E$ if there is a measurable set $A$ such that

$$
\begin{gathered}
\lim _{r \downarrow 0} \frac{\mathcal{L}^{n}\left((E \backslash A) \cap B_{r}(x)\right)}{r^{n}}=0 ; \\
\lim _{y \rightarrow x, y \in A} u(y)=u(x) .
\end{gathered}
$$

We recall, then, the following classical result in real analysis and its improved version for $B V$ functions (we refer to Section 3.7 of [1]).

Proposition 2.7. Measurable maps are approximately continuous a.e.. If u is a BV map of $n$ variables, then we can redefine it on a set of measure zero so to get a precise representative $\tilde{u}$ which is approximately continuous at every point $x$ which satisfies

$$
\lim _{r \downarrow 0} \frac{|D u|\left(B_{r}(x)\right)}{r^{n-1}}=0 .
$$

If $N$ denotes the set of points where (12) fails, then $\mathcal{H}^{n-1}\left(N \backslash J_{u}\right)=0$. Moreover, for every $x \in J_{u}$, there exist two distinct values $u^{+}(x)$ and $u^{-}(x)$ and a measurable set $G$ such that:

$$
\begin{aligned}
\lim _{r \downarrow 0} \frac{\mathcal{L}^{n}\left(B_{r}(x) \backslash G\right)}{r^{n}} & =0 ; \\
\lim _{y \rightarrow x, y \in G,\langle(y-x), \nu(x)\rangle<0} \tilde{u}(y) & =u^{-}(x) ; \\
\lim _{y \rightarrow x, y \in G,\langle(y-x), \nu(x)\rangle>0} \tilde{u}(y) & =u^{+}(x) .
\end{aligned}
$$

Finally, it is useful for our analysis that, roughly speaking, points of approximate continuity of traces of BV functions and points of approximate continuity of the functions themselves, coincide "most of the time". The precise statement is given below. We restrict ourselves to the case of 2-dimensional BV functions, which is the one really needed for our purposes. However, the statement can be suitably generalized to any dimensions.

Proposition 2.8. Let $u \in B V\left([0,1]^{2}\right)$ and consider the function $\tilde{u}$ of Proposition 2.7. Then, the following property holds for a.e. $y$ :

- If $(y, x) \notin J_{u} \cap(\{y\} \times[0,1])$, then

$$
\lim _{z \rightarrow x,(y, z) \notin J_{u}} \tilde{u}(y, z)=\tilde{u}(y, x) .
$$


Proof. First of all, consider the two sets of $y$ 's, $N_{1}$ and $N_{2}$ such that (1) of Theorem 2.3 apply. For each $y \in N_{2}$, let $G_{y}^{2}$ be the set of points $y$ of approximate continuity of $u(\cdot, y)$ and set

$$
G^{2}:=\cup_{t} G_{t}^{2} \times\{t\}
$$

Finally, let $N$ be the set of Proposition 2.7 and recall that $\mathcal{H}^{1}\left(N \backslash J_{u}\right)=0$.

We are now ready to give the set of $y$ 's for which the conclusion of the Proposition holds. More precisely, $y$ has to satisfy the following conditions:

(c1) $y \in N_{1}$ and $(\{y\} \times[0,1]) \cap\left(N \backslash J_{u}\right)=\emptyset$;

(c2) $(y, x) \in G^{2}$ for a.e. $x \in[0,1]$.

Fix a $y$ satisfying the two conditions above and an $x$ with $(y, x) \notin J_{u}$. We claim that

$(\mathrm{Cl}) v(\cdot):=\widetilde{u}(y, \cdot)$ is approximately continuous at any such $x$.

Assume for the moment that $(\mathrm{Cl})$ holds. By the classical properties of $1 \mathrm{~d}$ BV functions (see Section 2.2), after redefining $v$ on a set of measure zero, we get a new $\tilde{v}$ which is continuous at every $x \notin J_{u}$. On the other hand, we must have $v(x)=\tilde{v}(x)$ at every point where $v$ is approximately continuous. So, after having proved $(\mathrm{Cl})$, we conclude that $\tilde{v}$ and $\tilde{u}(y, \cdot)$ coincide at every point $x$ with $(y, x) \notin J_{u}$. This proves the proposition.

It remains to show $(\mathrm{Cl})$. We argue by contradiction and assume it is false. Then at some $x$ with $(y, x) \notin J_{u}$, we have a constant $\eta>0$ with the following property. If we define

$$
A_{r}:=\{z \in] x-r, x+r[:|\tilde{u}(y, z)-\tilde{u}(y, x)| \geq \eta\},
$$

then

$$
\limsup _{r \downarrow 0} \frac{\mathcal{L}^{1}\left(A_{r}\right)}{r} \geq \eta
$$

Now, set $A_{r}^{\prime}:=\left\{z \in A_{r}:(y, z) \in G^{2}\right\}$. By $(\mathrm{c} 2) \mathcal{L}^{1}\left(A_{r} \backslash A_{r}^{\prime}\right)=0$. We further restrict $A_{r}^{\prime}$ by setting $A_{r}^{\prime \prime}:=\left\{z \in A_{r}^{\prime}:(\tau, z) \in G^{2}\right.$ for a.e. $\left.\tau\right\}$. Then, by Fubini, $\mathcal{L}^{1}\left(A_{r}^{\prime} \backslash A_{r}^{\prime \prime}\right)=0$. Hence

$$
\limsup _{r \downarrow 0} \frac{\mathcal{L}^{1}\left(A_{r}^{\prime \prime}\right)}{r} \geq \eta
$$

On the other hand, for $z \in A_{r}^{\prime \prime}$, (recalling that $\left.(y, z) \in G^{2}\right)$ we can write

$$
|\tilde{u}(\tau, z)-\tilde{u}(y, z)| \leq\left|\frac{d}{d t} u(\cdot, z)\right|(] y-r, y+r[)=: g(r, z)
$$

for every $\tau \in] y-r, y+r\left[\in G^{2}\right.$ (and hence for a.e. $\left.\tau \in\right] y-r, y+r[$ ). Since, by $(\mathrm{c} 1)$, $(y, x) \notin N$, we know that

$$
\lim _{r \downarrow 0} \frac{1}{r} \int_{x-r}^{x+r} g(r, z) d z \leq \lim _{r \downarrow 0} \frac{|D u|\left(B_{2 r}(y, x)\right)}{r}=0 .
$$

So, for the set

$$
C_{r}:=A_{r}^{\prime \prime} \cap\{z: g(r, z)<\eta / 2\}
$$


we have

$$
\lim _{r \downarrow 0} \frac{\mathcal{L}^{1}\left(A_{r}^{\prime \prime} \backslash C_{r}\right)}{r}=0, \quad \text { which implies } \quad \limsup _{r \downarrow 0} \frac{\mathcal{L}^{1}\left(C_{r}\right)}{r} \geq \eta .
$$

Consider finally the set $D_{r}:=\left\{(\tau, z): z \in C_{r},|\tau-y|<r\right\} \cap G^{2}$. It turns out that:

- $\lim \sup _{r \downarrow 0} r^{-2}\left|D_{r}\right| \geq \eta / 2$;

- $D_{r} \subset B_{2 r}((y, x))$;

- If $(\tau, z) \in D_{r}$, then

$$
|\tilde{u}(\tau, z)-\tilde{u}(y, x)| \geq|\tilde{u}(y, z)-\tilde{u}(y, x)|-|\tilde{u}(\tau, z)-\tilde{u}(y, z)| \geq \eta-\frac{\eta}{2}=\frac{\eta}{2} .
$$

The existence of the sets $D_{r}$ obviously contradict the approximate continuity of $\tilde{u}$ at $(y, x)$, which must hold because $(y, x) \notin N$.

Proof of Proposition 2.1. Consider for any $t$ the SBV map $u_{t}$. Consider now the precise representative $\widetilde{u_{t}}$ of $u_{t}$, given by Proposition 2.7. $\widetilde{u_{t}}$ and $u_{t}$ differ on a set of measure zero $L_{t}$. Moreover, $\widetilde{u}_{t}$ is approximately continuous at all points $x$ for which

$$
\lim _{r \downarrow 0} \frac{\left|D u_{t}\right|\left(B_{r}(x)\right)}{r}=0 .
$$

On the other hand, by the definition of $\mathcal{S}^{\gamma}$, we have $D u_{t}=\nabla u_{t} \mathcal{L}^{2}+f \nu \mathcal{H}^{1}\llcorner\gamma(t)$. Now, since $0 \leq u_{t} \leq t$ a.e., it is a standard fact that $|f| \leq t$. Moreover, since $H\left(x, \nabla u_{t}(x)\right) \leq 0$ for a.e. $x$, assumption (H3) implies that $\left|\nabla u_{t}(x)\right| \leq \lambda^{-1}$. Thus $\left|D u_{t}\right| \leq \lambda^{-1} \mathcal{L}^{2}+t \mathcal{H}^{1}\llcorner\gamma(t)$ and, if (21) fails, we necessarily have

$$
\limsup _{r \downarrow 0} \frac{\mathcal{H}^{1}\left(\gamma(t) \cap B_{r}(x)\right)}{r}>0 .
$$

The completeness of $\gamma$, implies that:

$$
\widetilde{u}_{t} \text { is approximately continuous at every } x \notin \gamma(t) \text {. }
$$

Obviously, if $t<\tau$, then $\widetilde{u_{t}}(x) \leq \widetilde{u_{\tau}}(x)$ for a.e. $x$. Moreover, if $x$ is a point of approximate continuity of $\widetilde{u_{t}}$ and $\widetilde{u_{t}}(x)<t$, then

(a) $x$ is a point of approximate continuity for $\widetilde{u_{\tau}}$ for every $\tau$;

(b) $\widetilde{u_{\tau}}(x)=\widetilde{u_{t}}(x)$ for every $\tau>t$ and $\widetilde{u_{\tau}}(x) \leq \widetilde{u_{t}}(x)$ for every $\tau \leq t$.

Set then $\widetilde{u}(x):=\sup _{t} \widetilde{u_{t}}(x)$.

Step 1 First we prove assertion (i), that is $\tilde{u}=u$ a.e.. Indeed, consider first the set $A_{N}:=\{\tilde{u}<N\}$, where $N \in \mathbb{N}$. Then $\tilde{u}=\widetilde{u_{N}}$ on the set $A_{N}^{\prime} \subset A_{N}$ of points of approximate continuity for $\widetilde{u_{N}}$ and $\tilde{u}$. Indeed, at such a point $x$ we have $\widetilde{u_{N}}(x) \leq \tilde{u}(x)<N$. Thus we can apply (a) and (b), from which we conclude $\tilde{u}(x)=\sup _{\tau} \widetilde{u_{\tau}}(x)=\widetilde{u_{N}}(x)$. Observe next that $\left|A_{N} \backslash A_{N}^{\prime}\right|=0$ and that $\widetilde{u_{N}}=u_{N}$ on a set $A_{N}^{\prime \prime} \subset A_{N}^{\prime}$ with $\left|A_{N}^{\prime} \backslash A_{N}^{\prime \prime}\right|=0$. On the other hand, on every $x \in A_{N}^{\prime \prime}$ we have $u_{N}(x)<N$ and thus $u(x)=u_{N}(x)=\widetilde{u_{N}}(x)=\tilde{u}(x)$. So, $u=\tilde{u}$ a.e. on $A_{N}$.

Since $\cup_{N} A_{N}=\{\tilde{u}<\infty\}$, it remains to show that $u=\infty$ a.e. on $A:=\{\tilde{u}=\infty\}$. Consider now the subset $A^{\prime} \subset A$ of points $x$ where all $\widetilde{u_{N}}$ are approximately continuous. 
Clearly $\left|A \backslash A^{\prime}\right|=0$. On the other hand, on each $x \in A^{\prime}$ we necessarily have $\widetilde{u_{N}}(x)=N$. Otherwise, by (a) and (b) we would have $\tilde{u}(x)=\sup _{\tau} \widetilde{u_{\tau}}(x)=\widetilde{u_{N}}(x)<N$, contradicting $\tilde{u}(x)=\infty$. Consider next the set $A^{\prime \prime} \subset A^{\prime}$ of points $x$ where $\widetilde{u_{N}}(x)=u_{N}(x)$ for every $N$. Again $\left|A^{\prime} \backslash A^{\prime \prime}\right|=0$. Hence, for every $x \in A^{\prime \prime}$ we have $u_{N}(x)=\widetilde{u_{N}}(x)=N$. Letting $N \uparrow \infty$ we conclude $u(x)=\infty$ for every $x \in A^{\prime \prime}$.

Step 2 We claim next that, if $\widetilde{u_{t}}$ is approximately continuous at $x$, so is $\tilde{u}_{t}$ (observe that $\widetilde{u_{t}}$ is the precise representative of $u_{t}$, whereas $\tilde{u}_{t}=\tilde{u} \wedge t$ ). Assume indeed that $\widetilde{u_{t}}$ is approximately continuous at $x$. Let then $E$ be a measurable set satisfying the requirements of Definition 2.6. Obviously, if we reduce further $E$ taking all the points $y \in E$ of approximate continuity for $\widetilde{u_{t}}$, the new set still satisfies the requirements of Definition 2.6. With a slight abuse of notation, we keep the name $E$ for this second set. Next, if $y \in E$, either $\widetilde{u_{t}}(y)<t$, and hence $\tilde{u}(y)=\widetilde{u}_{t}(y)$ (because $\widetilde{u_{t}}$ is approximately continuous at $y$ and hence (b) applies), or $\widetilde{u_{t}}(y)=t$ and hence $\tilde{u}(y) \geq t$. In both cases, $\tilde{u}_{t}(y)=\widetilde{u}_{t}(y)$. For the same reasons $\tilde{u}_{t}(x)=\widetilde{u}_{t}(x)$. We therefore conclude that

$$
\lim _{y \in E, y \rightarrow x} \tilde{u}_{t}(y)=\lim _{y \in E, y \rightarrow x} \tilde{u}_{t}(y)=\widetilde{u}_{t}(x)=\tilde{u}_{t}(x) .
$$

This shows that all the points of approximate continuity of $\widetilde{u_{t}}$ are points of approximate continuity of $\tilde{u}_{t}$. Thus assertion (ii) follows from (23). Finally, assertion (iii) follows easily from Proposition 2.8, Theorem 2.3 and assertion (ii).

\section{Zig-ZAG CONSTRUCTION AND FASTER TRAJECTORIES}

3.1. Zig-zag constructions. In this section we outline a crucial construction for our proof of Theorem 1.5. The basic idea is borrowed from [7], but we require several technical improvements. We assume that

(Z1) $\gamma$ is an admissible strategy, not necessarily complete;

(Z2) $t \in] 0, \infty\left[\right.$ and $x_{0}$ is a point such that

$$
\lim _{r \downarrow 0} \frac{\mathcal{H}^{1}\left(B_{r}\left(x_{0}\right) \cap \gamma(t)\right)}{r}=0 .
$$

Lemma 3.1 (Zig-zag). Assume (Z1)-(Z2) and let $\varepsilon$ be any given positive number. Then there is a set $G$ of radii such that

$$
\lim _{r \downarrow 0} \frac{\mathcal{L}^{1}([0, r] \backslash G)}{r}=0
$$

and the following property holds.

If $B_{\varepsilon}(v) \subset F\left(x_{0}\right), \mu|v| \in G$ and $\tau<t-\mu$, then there exists a Lipschitz trajectory $z:[\tau, \tau+\mu] \rightarrow \mathbb{R}^{2}$ satisfying the following assumptions

(z1) $z(\tau)=x_{0}, z(\tau+\mu)=x_{0}+\mu v$;

(z2) $\dot{z}(s) \in F(z(s))$ for a.e. $s$;

(z3) $z(s) \notin \gamma(t)$ for every $s$.

Assume in addition that $\gamma$ is a complete strategy, $u \in \mathcal{S}^{\gamma}$ and $\tilde{u}$ is the function given by Proposition 2.1. Then, we can require the following additional property: 
(z4) $w(s):=\tilde{u}_{t}(z(s))$ is Lipschitz, $u_{t}$ is approximately differentiable at $z(s)$ for a.e. $s$ and the following identities hold:

$$
\left\{\begin{array}{l}
\dot{w}(s)=\nabla u_{t}(z(s)) \cdot \dot{z}(s) \\
H\left(z(s), \nabla u_{t}(z(s))\right) \leq 0
\end{array} .\right.
$$

For $v$ and $\mu$ as above and $\tau<t$ there exists a trajectory $z:[\tau-\mu, \tau] \rightarrow \mathbb{R}^{2}$ enjoying (z2) $-(z 4)$ but with $z(\tau-\mu)=x_{0}-\mu v$ and $z(\tau)=x_{0}$.

Proof. The proof of the first assertion of the Theorem follows essentially from the same arguments proving the second assertion. We assume therefore that the strategy $\gamma$ is complete and prove the existence of a set $G$ satisfying (25) (and of the corresponding trajectories satisfying (z1)-(z4)).

Without loss of generality we assume $v=(1,0)$ and $x_{0}=0$. Observe also that (by the continuity of the multifunction $F$ ) there is a $\delta>0$ such that:

$$
B_{\varepsilon / 2}((\cos \theta, \sin \theta)) \subset F(x) \quad \text { if }|x|<\delta \text { and }|\theta| \leq \delta .
$$

By the properties of $\tilde{u}$, we know that $\tilde{u}_{t}$ is approximately continuous at 0 . Let therefore $A$ be a measurable set such that

(AC1) $r^{-2}\left|B_{r} \backslash A\right| \rightarrow 0$ for $r \downarrow 0$;

$(\mathrm{AC} 2) \tilde{u}_{t}(x) \rightarrow \tilde{u}_{t}(0)$ if $x \in A$ and $x \rightarrow 0$.

Next, fix a small positive number $\alpha<\delta$ to be chosen later. For every $r$ consider the arc of circle $\eta_{r}:=\{r(\cos \theta, \sin \theta):|\theta| \leq \alpha\}$. We denote by $H$ the set of radii $r$ such that $\gamma(t) \cap \eta_{r}=\emptyset$. By (Z2) it easily follows that

$$
\lim _{r \downarrow 0} \frac{\mathcal{L}^{1}([0, r] \backslash H)}{r}=0 .
$$

On the other hand, by Proposition 2.1 we can conclude that, for a.e. $r \in H$ :

(G1) $w=\left.\tilde{u}_{t}\right|_{\eta_{r}}$ is Lipschitz;

(G2) the derivative of $w$ at $p \in \eta_{r}$ is the tangential component of $\nabla u_{t}(p)$ for $\mathcal{H}^{1}$-a.e. $p \in \eta_{r}$

(G3) $H\left(p, \nabla u_{t}(p)\right) \leq 0$ for $\mathcal{H}^{1}$-a.e. $p \in \eta_{r}$.

We define $G$ as the set of elements $r \in H$ which satisfy (G1)-(G3) and which are smaller than a positive constant $c_{0}$ (to be chosen later). Then (25) holds. Next, for every $N \in \mathbb{N}$ and any angle $\theta \in]-\alpha, \alpha[$ consider the segment

$$
\sigma_{\theta, N}:=\left\{\rho(\cos \theta, \sin \theta): 2^{-(N+2)} \leq \rho \leq 2^{-N}\right\} .
$$

We say that $(\theta, N)$ is good if

(G4) The conditions corresponding to (G1)-(G3) are satisfied for $\left.\tilde{u}\right|_{\sigma_{\theta, N}}$;

(G5) There is a $\rho=\rho(N, \theta)$ between $\frac{3}{8} 2^{-N}$ and $2^{-N-1}$ such that

$$
\rho(N, \theta)(\cos \theta, \sin \theta) \in A .
$$


Obviously, again by (Z2) and by (AC1), there is a constant $c_{0}$ such that, for every $N$ with $2^{-N} \leq c_{0}$ there always exists an angle $\theta_{N}$ for which $\left(\theta_{N}, N\right)$ is good.

It is also easy to conclude that, by possibly choosing $c_{0}$ smaller, there is always a radius $\left.r_{N} \in\right] 2^{-(N+2)}, \frac{3}{8} 2^{-N}$ [ belonging to $H$. Assume therefore that $\mu \in G$. Let $N_{0}$ be the largest natural number such that $2^{-N_{0}} \geq \mu$. We construct a piecewise smooth curve joining $\mu(1,0)$ and $(0,0)$ as follows.

- We first let $p_{0}$ be the intersection of $\sigma_{\theta_{N_{0}}, N_{0}}$ with the arc $\eta_{\mu}$ and we let $\psi_{0}$ be the arc contained in $\eta_{\mu}$ joining $\mu(1,0)$ and $p_{0}$.

- We then let $q_{0}:=\sigma_{\theta_{N_{0}}, N_{0}} \cap \eta_{r_{N_{0}}}$ and denote by $\sigma_{0}$ the segment with endpoints $p_{0}$ and $q_{0}$;

- We let $p_{1}:=\sigma_{\theta_{N_{0}+1}, N_{0}+1} \cap \eta_{r_{N_{0}}}$ and let $\psi_{1}$ be the arc contained in $\eta_{r_{N_{0}}}$ joining $q_{0}$ and $p_{1}$.

We proceed inductively. The trajectory consists of infinitely many radial segments $\sigma_{i}$ and of infinitely many arcs $\psi_{i}$. We call their union $\Psi$. The sum the lengths of $\sigma_{i}$ is exactly $\mu$. The sum of the lengths of $\psi_{i}$ is bounded from above by $C \alpha \mu$, where $C$ is a geometric constant independent of $\alpha$ and $\mu$. We can go at all speeds up to $1+\varepsilon / 2$ along the segments $\sigma_{i}$ (by (27)) and at all speeds up to $\lambda$ along the $\operatorname{arcs} \psi_{i}$ (by (H3)).

Therefore, it is surely possible to go along the trajectory $\Psi$ with a map $z:[\tau, \tau+\mu] \rightarrow \Psi$ satisfying (z1) and (z2) if the following inequality holds:

$$
\mu\left(1+\frac{\varepsilon}{2}\right)^{-1}+C \alpha \frac{\mu}{\lambda} \leq \mu .
$$

However, this is certainly the case if $\alpha$ is chosen sufficiently small. Next, since $\Psi \cap \gamma(t)=\emptyset$, $z$ obviously satisfies (z3).

Now, the function $w=\tilde{u}_{t} \circ z$ is obviously locally Lipschitz on $\left.] \tau, \tau+\mu\right]$ because of $(\mathrm{G} 1)$ (G4). Moreover, (26) is satisfied, and therefore the Lipschitz constant of $w$ on any interval $[\tau+\nu, \tau+\mu]$ is bounded by a constant $C$ independent of $\nu>0$ (recall indeed that, by (H3), if $H(x, p) \leq 0$, then $\left.|p| \leq \lambda^{-1}\right)$. This means that $w$ extends to a continuous function $\tilde{w}$ on $[\tau, \tau+\mu]$ and, in order to conclude the proof, it suffices to check that $\tilde{w}(\tau)=w(\tau)$. Note that by our construction, the points $\rho\left(i, \theta_{i}\right)\left(\cos \theta_{i}, \sin \theta_{i}\right)$ belong to the trajectory $\Psi$ and they are hence equal to $z\left(\tau_{i}\right)$ for some sequence $\tau_{i} \downarrow \tau$. But then $z\left(\tau_{i}\right) \in A$, and by (AC2), we have that $w\left(\tau_{i}\right)=\tilde{u}_{t}\left(z\left(\tau_{i}\right)\right)$ converges to $\tilde{u}_{t}(0)=w(\tau)$. This completes the proof.

3.2. Faster trajectories. The last technical tool of the paper comes again from an idea of [7] (cp. to Lemma 7.1 therein). The obvious proof is left to the reader.

Lemma 3.2 (Faster trajectory). Let $x:[0, T] \rightarrow \mathbb{R}^{2}$ be an admissible trajectory, i.e.:

- $\dot{x}(t) \in F(x(t))$ for a.e. $t$;

- $x(t) \notin \gamma(t)$ for every $t$;

- $x(0) \in R_{0}$.

Let $0<\varepsilon<\delta$ and consider the trajectory $x^{\sharp}:[0, T-\varepsilon] \rightarrow \mathbb{R}^{2}$ given by

$$
x^{\sharp}(t)=x\left(\frac{T}{T+\delta+\varepsilon}(t+\delta+2 \varepsilon)\right) .
$$




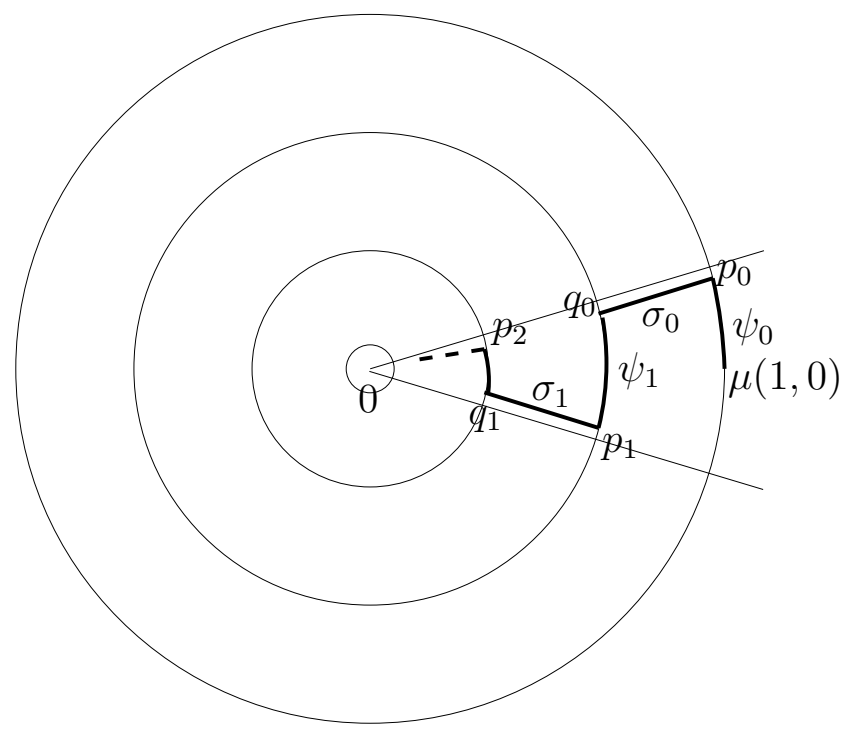

FiguRE 1. The zig-zag curve constructed in the proof of Lemma 3.1.

For $\delta$ and $\varepsilon$ appropriately small, we have

- $B_{2 \varepsilon}\left(\dot{x}^{\sharp}(t)\right) \subset F\left(x^{\sharp}(t)\right)$ for a.e. $t$;

- $x^{\sharp}(t) \notin \gamma(t+\varepsilon)$ for every $t$;

- $x^{\sharp}(0) \in R_{0}$.

\section{Proof of Theorem 1.5: Part I}

In this section we prove that $T^{\gamma}$ belongs to $\mathcal{S}^{\gamma}$ under the only assumption that $\gamma$ is an admissible strategy. Thus we have to show that $T^{\gamma}$ satisfies the requirements (a) and (b) of Definition 1.4.

4.1. Condition (a). Obviously $T^{\gamma} \equiv 0$ on $R_{0}$.

Step 1 We fix $t>0$ and start by showing that $T_{t}^{\gamma}$ belongs to $S B V_{l o c}$. For an arbitrary $x \in \mathbb{R}$, we set $l_{x}:=\{(x, y): y \in \mathbb{R}\}$ and $l_{x, \gamma}:=l_{x} \cap \gamma(t)$. We claim that

(Cl) $T_{t}^{\gamma}$ is locally Lipschitz on the interior of $l_{x} \backslash l_{x, \gamma}$, with Lipschitz constant smaller than $\lambda^{-1}$ (where $\lambda$ is the constant in (H3)).

We will prove this claim later. Obviously the same proof gives the following symmetric statement, where $l_{y}^{\prime}:=\{(x, y): x \in \mathbb{R}\}$ and $l_{y, \gamma}^{\prime}=l_{y}^{\prime} \cap \gamma(t)$ :

$\left(\mathrm{Cl}^{\prime}\right) T_{t}^{\gamma}$ is locally Lipschitz on the interior of $l_{y}^{\prime} \backslash l_{y, \gamma}^{\prime}$ with constant smaller than $\lambda^{-1}$.

First of all, $(\mathrm{Cl})$ and $\left(\mathrm{Cl}^{\prime}\right)$ imply the measurability of $T_{t}^{\gamma}$. Indeed, recall that $\gamma$ is rectifiable and hence Borel measurable. Therefore, for every fixed integer $j>0$ it is possible to find a closed set $\Gamma^{j} \subset \gamma(t)$ such that $\mathcal{H}^{1}\left(\gamma(t) \backslash \Gamma^{j}\right)<\frac{1}{j}$. Let $V_{j}, H_{j} \subset \mathbb{R}$ be the projections of the set $\gamma(t) \backslash \Gamma^{j}$ respectively on the horizontal and the vertical axis. $(\mathrm{Cl})$ and $\left(\mathrm{Cl}^{\prime}\right)$ imply 
that $T_{t}^{\gamma}$ is locally Lipschitz on

$$
C_{j}:=\left[\left(\left(\mathbb{R} \backslash H_{j}\right) \times \mathbb{R}\right) \cap\left(\mathbb{R} \times\left(\mathbb{R} \backslash V_{j}\right)\right)\right] \backslash \Gamma^{j} .
$$

Indeed, fix $\left(x_{1}, y_{1}\right) \in C_{j}$. Since $\Gamma^{j}$ is closed, there is a ball $B$ centered at $\left(x_{1}, y_{1}\right)$ such that $B \cap \Gamma^{j}=\emptyset$. Consider any other point $\left(x_{2}, y_{2}\right) \in B$ and let $\sigma$ and $\eta$ be the segments joining, respectively, $\left(x_{1}, y_{1}\right)$ with $\left(x_{1}, y_{2}\right)$ and $\left(x_{1}, y_{2}\right)$ with $\left(x_{2}, y_{2}\right)$. Since $x_{1} \notin H_{j}$ and $y_{2} \notin V_{j}$, the intersections $\eta \cap \gamma(t)$ and $\sigma \cap \gamma(t)$ must be contained in $\Gamma^{j}$. On the other hand the segments $\sigma$ and $\eta$ are also contained in $B$ and thus we conclude that $\eta \cap \gamma(t)=\sigma \cap \gamma(t)=\emptyset$. Therefore $(\mathrm{Cl})$ and $\left(\mathrm{Cl}^{\prime}\right)$ imply that

$$
\left|T_{t}^{\gamma}\left(x_{1}, y_{1}\right)-T_{t}^{\gamma}\left(x_{2}, y_{2}\right)\right| \leq \frac{\left|x_{1}-x_{2}\right|+\left|y_{1}-y_{2}\right|}{\lambda}
$$

Observe next that $\mathcal{L}^{1}\left(H_{j}\right)+\mathcal{L}^{1}\left(V_{j}\right)<2 / j$. Thus, $\mathbb{R}^{2} \backslash \bigcup C_{j}$ has zero Lebesgue measure and, having concluded that $T_{t}^{\gamma}$ is locally Lipschitz on each set $C_{j}$, we infer that $T_{t}^{\gamma}$ is measurable.

Note that, if $l_{x, \gamma}$ is finite, $(\mathrm{Cl})$ clearly implies that the restriction $\left.T_{t}^{\gamma}\right|_{l_{x}}$ is an SBV function with finitely many jumps. On the other hand we have the coarea formula

$$
\int \sharp\left(l_{x, \gamma}\right) d x \leq \mathcal{H}^{1}(\gamma(t))<\infty,
$$

which implies that $\left(l_{x, \gamma}\right)$ is finite for a.e. $x$. Since $0 \leq T_{t}^{\gamma} \leq t$, each jump has size at most $t$ and we therefore bound

$$
\int_{-R}^{R}\left\|\frac{d}{d y} T_{t}^{\gamma}(x, \cdot)\right\|_{T V(]-R, R[)} d x \leq \int_{-R}^{R}\left(\lambda^{-1}+t \sharp\left(l_{x, \gamma}\right)\right) d x \stackrel{(29)}{<}+\infty .
$$

The same argument applies if we fix the $y$ coordinate and let $x$ vary. We can therefore apply Theorem 2.3 to conclude that $T_{t}^{\gamma} \in S B V(]-R, R\left[^{2}\right)$ for every positive $R$. This shows that $T_{t}^{\gamma} \in S B V_{l o c}$.

We now come to the proof of $(\mathrm{Cl})$. We fix $Y=(x, y) \in l_{x} \backslash l_{x, \gamma}$ and distinguish two cases:

Case 1: $\tau:=T_{t}^{\gamma}(x, y)<t$. In this case $T_{t}^{\gamma}(x, y)=T^{\gamma}(Y)$. We fix $\varepsilon<\frac{t-\tau}{2}$ and

$$
\delta<\min \left\{\varepsilon, \lambda^{-1} \operatorname{dist}\left((x, y), l_{x, \gamma}\right)\right\} .
$$

Let $Z=(x, z)$. When $|z-y|<\delta$ we consider the path $\varphi:\left[0, \lambda^{-1}|z-y|\right] \rightarrow \mathbb{R}^{2}$ given by

$$
\varphi(s)=\left(x, y+\frac{z-y}{|z-y|} \lambda s\right)=Y+\frac{Z-Y}{|Z-Y|} \lambda s .
$$

It is easy to see that $\dot{\varphi} \in F(\varphi)$ (because of (H3)) and that $\varphi(s) \notin \gamma(t)$. On the other hand, if $T$ is a given time in $] \tau, \tau+\varepsilon\left[\right.$, there is an admissible path $\psi:[0, T] \rightarrow \mathbb{R}^{2}$ which starts from a point $\psi(0) \in R_{0}$ and reaches $Y=(x, y)$. If we join the paths $\psi$ and $\varphi$ in the obvious 
way, then we obtain an admissible path which reaches $Z=(x, z)$ at a time $T+\lambda^{-1}|z-y|$. Since $T$ can be chosen arbitrarily close to $\tau=T^{\gamma}(x, y)$, we conclude

$$
T^{\gamma}(x, z) \leq T^{\gamma}(x, y)+\frac{1}{\lambda}|z-y| .
$$

On the other hand, a symmetric argument shows

$$
T^{\gamma}(x, z) \geq T^{\gamma}(x, y)-\frac{1}{\lambda}|z-y|
$$

which therefore completes the proof of the claim.

Case $2: T^{\gamma}(x, y) \geq t$. In this case $T_{t}^{\gamma}(x, y)=t$ and, since $T_{t}^{\gamma} \leq t$, it suffices to show

$$
T^{\gamma}(x, z) \geq t-\lambda^{-1}|z-y|
$$

for any $z$ sufficiently close to $y$. On the other hand, if (34) were false for a sufficiently close $z$, we could argue as in (32) reversing the roles of $z$ and $y$ and finding

$$
T^{\gamma}(x, y) \leq T^{\gamma}(x, z)+\lambda^{-1}|z-y|<t,
$$

which contradicts our assumption $T^{\gamma}(x, y) \geq t$.

Step 2 To complete the proof that (a) in Definition 1.4 is satisfied, we must show that the jump set $J$ of $T_{t}^{\gamma}$ is contained in $\gamma(t)$. Let $A$ be the set of $x$ 's such that $\sharp l_{x, \gamma}<\infty$ and $B$ the set of $y$ 's for which $\sharp l_{y, \gamma}^{\prime}<\infty$. In the previous subsection we have shown that $\mathcal{L}^{1}(\mathbb{R} \backslash A)=0$ and that for any $x \in A$ the jump set $J_{x}$ of $\left.T_{t}^{\gamma}\right|_{l_{x}}$ is contained in $\gamma(t)$. By Theorem 2.3, there is a further set $A^{\prime} \subset A$ with $\mathcal{L}^{1}\left(A \backslash A^{\prime}\right)=0$ such that $J_{x}=J \cap l_{x}$ for every $x \in A^{\prime}$. We thus conclude that $J \cap\left(A^{\prime} \times \mathbb{R}\right) \subset \gamma(t)$ and $\mathcal{L}^{1}\left(\mathbb{R} \backslash A^{\prime}\right)=0$. Arguing similarly for the $y$ coordinates, we conclude the existence of a set $B^{\prime}$ with $\mathcal{L}^{1}\left(\mathbb{R} \backslash B^{\prime}\right)=0$ such that

$$
J \subset \gamma(t) \cup\left(\left(\left(\mathbb{R} \backslash A^{\prime}\right) \times \mathbb{R}\right) \cap\left(\mathbb{R} \times\left(\mathbb{R} \backslash B^{\prime}\right)\right)\right) .
$$

On the other hand $\left(\left(\left(\mathbb{R} \backslash A^{\prime}\right) \times \mathbb{R}\right) \cap\left(\mathbb{R} \times\left(\mathbb{R} \backslash B^{\prime}\right)\right)\right)=\left(\mathbb{R} \backslash A^{\prime}\right) \times\left(\mathbb{R} \backslash B^{\prime}\right)$. But, since $J$ is a 1 -d rectifiable set, $\mathcal{H}^{1}\left(J_{T_{t}^{\gamma}} \cap\left(\left(\mathbb{R} \backslash A^{\prime}\right) \times\left(\mathbb{R} \backslash B^{\prime}\right)\right)=0\right.$.

4.2. Condition (b). We start by observing that (5) holds a.e. on $\left\{T_{t}^{\gamma}=t\right\}$. Indeed, if this set has measure zero, then there is nothing to prove. Otherwise, using Theorem 2.2 and the Lebesgue Theorem it is easy to show that $\nabla T_{t}^{\gamma}=0$ a.e. on $\left\{T_{t}^{\gamma}=t\right\}$. Since (H3) implies that $H(X, 0)<0$ for every $X$, this proves our claim. The same observation shows that (5) holds at every $X \in R_{0}$.

We fix next a point $X$ such that

- $T^{\gamma}(X)=T_{t}^{\gamma}(X)<t$

- $T_{t}^{\gamma}$ is approximately differentiable with differential $\nabla T_{t}^{\gamma}(X)$;

- $X \notin \bar{R}_{0}$ and

$$
\lim _{r \downarrow 0} \frac{\mathcal{H}^{1}\left(\gamma(t) \cap B_{r}(X)\right)}{r}=0
$$


Clearly, a.e. $X \in \mathbb{R}^{2} \backslash\left(R_{0} \cup\left\{T_{t}^{\gamma}=t\right\}\right)$ satisfies these requirements. Our aim is to show

$$
\nabla T_{t}^{\gamma}(X) \cdot w \leq 1, \quad \text { for every } w \in F(\stackrel{\circ}{X}) .
$$

From this easily follows that:

$$
H\left(X, \nabla T_{t}^{\gamma}(X)\right)=\sup _{w \in F(X)} \nabla T_{t}^{\gamma}(X) \cdot w-1 \leq 0 .
$$

We now show (37) and fix, therefore, $w \in F(X)$. Choose $\varepsilon \in] 0,1 / 2\left[\right.$ so that $B_{2 \varepsilon}(w) \subset$ $F(X)$ and $T^{\gamma}(X)+2 \varepsilon<t$. Apply Lemma 3.1 with $x_{0}=X, t, \varepsilon$ and $u=T^{\gamma}$. Let $\tau \in] T^{\gamma}(X), T^{\gamma}(X)+\varepsilon\left[\right.$ and $v$ a vector in $B_{\varepsilon}(w)$. $G$ is the set given by Lemma 3.1. If $\mu$ is such that $\mu|v| \in G$ and $\mu<\varepsilon$, let $z:[\tau, \tau+\mu]$ be the trajectory given by the first assertion of Lemma 3.1. Since $\tau \in] T^{\gamma}(X), T^{\gamma}(X)+\varepsilon\left[\right.$, there exists a trajectory $x:[0, \tau] \rightarrow \mathbb{R}^{2}$ such that

- $x(0) \in R_{0}, x(\tau)=X$;

- $\dot{x}(s) \in F(x(s))$ for a.e. $s$;

- $x(s) \notin \gamma(s)$ for every $s$.

Obviously, if we extend $x$ to $[0, \tau+\mu]$ by setting $x(s)=z(s)$ for $s \in[\tau, \tau+\mu], x$ continues to enjoy the same properties. This implies that $T^{\gamma}(X+\mu v)<\tau+\mu$. Let now $\tau$ converge to $T^{\gamma}(X)$ to conclude

$$
T_{t}^{\gamma}(X+\mu v) \leq T^{\gamma}(X+\mu v) \leq T^{\gamma}(X)+\mu=T_{t}^{\gamma}(X)+\mu .
$$

Since $T_{t}^{\gamma}$ is approximately differentiable at $X$, we find a set $B$ satisfying (i) and (ii) of Theorem 2.2. Clearly, for every $\eta>0$, there are $\mu<\eta$ and $v \in B_{\varepsilon}(w)$ such that $X+\mu v \in B$ and $\mu|v| \in G$.

We thus conclude that, for every $\varepsilon>0$ and $\kappa>0$, we find $\mu<\varepsilon$ and $v \in B_{\varepsilon}(w)$ such that

We thus can estimate

$$
\nabla T_{t}^{\gamma}(X) \cdot v \leq \frac{T_{t}^{\gamma}(X+\mu v)-T_{t}^{\gamma}(X)}{\mu}+\kappa \leq 1+\kappa
$$

$$
\begin{aligned}
\nabla T_{t}^{\gamma}(X) \cdot w & \leq \nabla T_{t}^{\gamma}(X) \cdot v+\left|\nabla T_{t}^{\gamma}(X)\right||w-v| \\
& \leq\left|\nabla T_{t}^{\gamma}(X)\right| \varepsilon+1+\kappa .
\end{aligned}
$$

Letting $\kappa$ and $\varepsilon$ go to 0 we conclude

$$
\nabla T_{t}^{\gamma}(X) \cdot w \leq 1
$$

\section{Proof of Theorem 1.5: Part II}

In this section we prove the second part of Theorem 1.5. We first claim that $\mathcal{S}^{\gamma}=\mathcal{S}^{\gamma^{c}}$. The inclusion $\mathcal{S}^{\gamma} \subset \mathcal{S}^{\gamma^{c}}$ is obvious. In order to show the opposite inclusion, recall that there is a countable set $C$ of $t$ 's such that $\mathcal{H}^{1}\left(\gamma^{c}(t) \backslash \gamma(t)\right)=0$ for every $t \notin C$. Thus, let $u \in \mathcal{S}^{\gamma^{c}}$. The only thing we need to show is that $\mathcal{H}^{1}\left(J_{u_{t}} \backslash \gamma(t)\right)=0$ for $t \in C$, since for $t \notin C$ this identity is trivial. Fix therefore a $t \in C$ and a point $x$ in $J_{u_{t}}$. Let $u_{t}^{-}(x)$ and $u_{t}^{+}(x)$ be the left and right approximate values of $u_{t}$ at $x$, according to Proposition 2.7. To fix 
ideas, assume $u_{t}^{+}(x)>u_{t}^{-}(x)$ (recall that the two values are necessarily different!). Then, for $\tau>u_{t}^{-}(x)$, we obviously conclude that $x$ is not a point of approximate continuity for $\tau$. Choose a sequence $\left\{\tau_{i}\right\} \subset \mathbb{R} \backslash C$ with $\tau_{i} \uparrow t$. According to Proposition 2.7, our argument shows

$$
\mathcal{H}^{1}\left(J_{u_{t}} \backslash \bigcup_{i} J_{u_{\tau_{i}}}\right)=0 .
$$

On the other hand $\mathcal{H}^{1}\left(J_{u_{\tau_{i}}} \backslash \gamma^{c}\left(\tau_{i}\right)\right)=0, \mathcal{H}^{1}\left(\gamma^{c}\left(\tau_{i}\right) \backslash \gamma\left(\tau_{i}\right)\right)=0$ and $\gamma\left(\tau_{i}\right) \subset \gamma(t)$. Therefore we conclude $\mathcal{H}^{1}\left(J_{u_{t}} \backslash \gamma(t)\right)=0$.

Having proved that $\mathcal{S}^{\gamma}=\mathcal{S}^{\gamma^{c}}$, we can assume that $\gamma$ itself is a complete strategy and aim at proving that $T^{\gamma}$ is the maximal element of $\mathcal{S}^{\gamma}$. Thus we consider an arbitrary $u \in \mathcal{S}^{\gamma}$ and, to simplify the notation, we assume that $u=\tilde{u}$, where $\tilde{u}$ is the function of Proposition 2.1. Our goal is to show that $u \leq T^{\gamma}$ a.e.. This condition is obvious on $R_{0}$ and on the set $\left\{T^{\gamma}=+\infty\right\}$. Thus, we can assume that

- $X \notin \bar{R}_{0}, X \notin \gamma_{\infty}, u$ is approximately continuous at $X$ and $T^{\gamma}(X)<\infty$.

We fix therefore such a point $X$ and we will show that, for every positive $\varepsilon, u(X) \leq$ $T^{\gamma}(X)+\varepsilon$.

Using Lemma 3.2 we can assume that, for some positive $T<T^{\gamma}(X)+\varepsilon$ and some $\delta>0$, there exists a trajectory $x:[0, T] \rightarrow \mathbb{R}^{2}$ such that

- $x(0) \in R_{0}$;

- $B_{2 \delta}(\dot{x}(t)) \subset F(x(t))$ for a.e. $t$;

- $x(t) \notin \gamma(t+\delta)$ for every $t$;

- $x(T)=X$.

We next define a set $\mathcal{P} \subset[0, T]: s$ belongs to $\mathcal{P}$ if and only if there is a trajectory $y:[0, s] \rightarrow \mathbb{R}^{2}$ with the following properties:

$(\mathrm{P} 1) y(0)=x(0)$ and $y(s)=x(s)$;

(P2) $\dot{y}(\sigma) \in F(y(\sigma))$ for a.e. $\sigma$;

(P3) $w:=u_{T+\delta} \circ y$ is Lipschitz and for a.e. $\sigma$ we have

$$
\text { either } \dot{w}(\sigma)=0 \text { or }\left\{\begin{array}{l}
u_{T+\delta} \text { is approximately differentiable at } y(\sigma) \\
\dot{w}(\sigma)=\nabla u_{T+\delta}(y(\sigma)) \cdot \dot{y}(\sigma) \\
H\left(y(\sigma), \nabla u_{T+\delta}(y(\sigma))\right) \leq 0
\end{array}\right\} \text {. }
$$

We will show below that:

- $\mathcal{P}$ has a maximal element;

- the maximal element of $\mathcal{P}$ is necessarily $T$.

We assume, for the moment, these two facts and conclude our proof. Since $T \in \mathcal{P}$, there is a trajectory $y:[0, T] \rightarrow \mathbb{R}^{2}$ satisfying (P1)-(P3). Note that, in a neighborhood of 0 , 
the trajectory $y$ takes values in $R_{0}$, where $u_{T+\delta}$ vanishes identically. Hence $w(0)=0$. Moreover, for a.e. $\sigma$, either $\dot{w}(\sigma)=0$ or

$$
\begin{aligned}
\dot{w}(\sigma) & =\nabla u_{T+\delta}(y(\sigma)) \cdot \dot{y}(\sigma) \leq \sup _{v \in F(y(\sigma))} \nabla u_{T+\delta}(y(\sigma)) \cdot v \\
& =1+H\left(y(\sigma), \nabla u_{T+\delta}(y(\sigma)) \leq 1 .\right.
\end{aligned}
$$

Therefore we conclude

$$
u_{T+\delta}(X)=w(T)=\int_{0}^{T} \dot{w}(\tau) d \tau \leq T .
$$

But this implies $u(X)=u_{T+\delta}(X)<T^{\gamma}(X)+\varepsilon$, which is the desired conclusion.

\section{Step 1. $\mathcal{P}$ has a maximal element.}

Let $S:=\sup \mathcal{P}$. If $x(S)=x(0)$, then the assertion is trivial. Therefore, without loss of generality, we assume $X:=x(S) \neq x(0)$. We let $\left\{s_{i}\right\}$ be a sequence in $\mathcal{P}$ converging to $S$ and we denote by $y_{i}$ the corresponding trajectories satisfying the conditions (P1)-(P3). The idea is that, for $i$ sufficiently large, we will be able to prolong the trajectory to reach $X$. This will be done by adding a zig-zag curve to a portion of $y_{i}$.

Next, we set

$$
a_{i}:=\frac{x(S)-x\left(s_{i}\right)}{S-s_{i}}
$$

and, passing to a subsequence, we assume that $a_{i}$ converges to some point. We set $a$ equal to this limit if it is different from 0 (we call this the principal case). If not, we distinguish two possibilities. If $x\left(s_{i}\right)=x(S)$ for some $i$, then we trivially have $S \in \mathcal{P}$. Indeed, it suffices to put $y(\tau)=y_{i}(\tau)$ for $\tau \leq s_{i}$ and $y(\tau)=x\left(s_{i}\right)=x(S)$ for $\tau \in\left[s_{i}, S\right]$ to get a trajectory $y$ satisfying (P1), (P2) and (P3). Otherwise, we can assume (passing to a subsequence) that

$$
\frac{x(S)-x\left(s_{i}\right)}{\left|x(S)-x\left(s_{i}\right)\right|}
$$

converges to some limit $\tilde{a}$ with $|\tilde{a}|=1$. In this case we set $a:=\lambda \tilde{a} / 2$ and we call it secondary case. It will be clear from the proof below that this situation is just a variant of the principal case. We therefore assume that $a \neq 0$ is the limit of the $a_{i}$ and leave to the reader the obvious modifications for the secondary case.

Note that, by our assumptions on $F$, it follows easily that $B_{2 \delta}(a) \subset F(x(S))$. Next choose $v=(1+\kappa) a$, where $\kappa$ is a positive constant, chosen so that $B_{\delta}(v) \subset F(x(S))$. To fix ideas, assume $a=(1,0)$ and $x(S)=0$. Fix moreover $\alpha>0$ (to be chosen later), set $\tau_{i}=S-s_{i}$ and consider, for every $i$ and for every $\left.\beta \in\right] \alpha / 2, \alpha\left[\right.$ the set $Q_{i, \beta}$ delimited by

- the segments

$$
d^{+}=\left[\tau_{i}(1-\beta)(\cos \beta, \sin \beta), \tau_{i}(1+\beta)(\cos \beta, \sin \beta)\right]
$$

and

$$
d^{-}=\left[\tau_{i}(1-\beta)(\cos \beta,-\sin \beta), \tau_{i}(1+\beta)(\cos \beta,-\sin \beta)\right]
$$


- the arcs $a r^{-}$and $a r^{+}$with radii, respectively, $\tau_{i}(1-\beta)$ and $\tau_{i}(1+\beta)$ and delimited, respectively, by the pair of points

$$
\tau_{i}(1-\beta)(\cos \beta,-\sin \beta) \quad \tau_{i}(1-\beta)(\cos \beta, \sin \beta)
$$

and by the pair of points

$$
\tau_{i}(1+\beta)(\cos \beta,-\sin \beta) \quad \tau_{i}(1+\beta)(\cos \beta, \sin \beta) .
$$

See Figure 2.

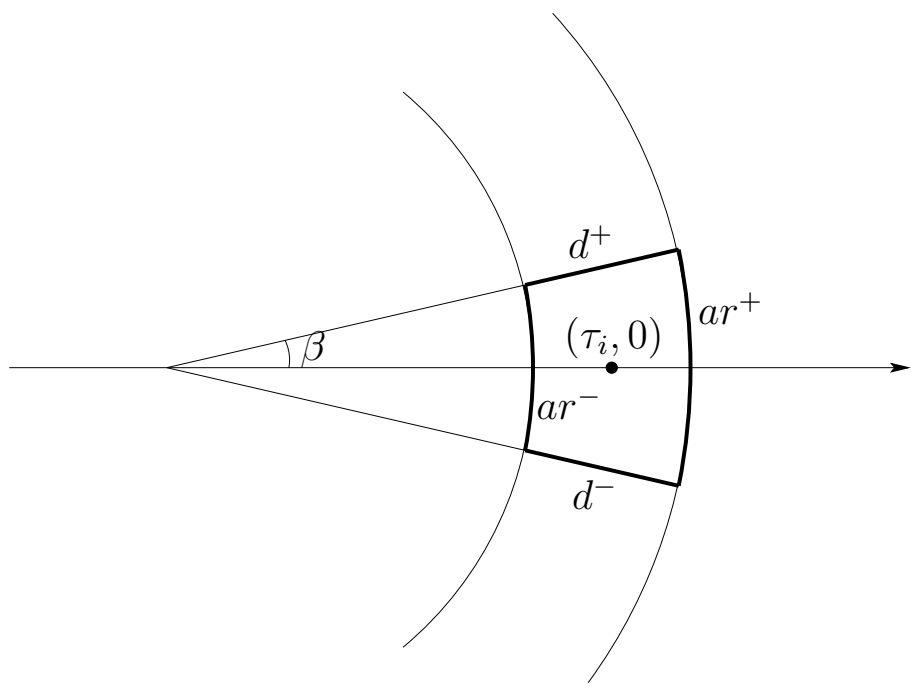

Figure 2. The set $Q_{i, \beta}$

Observe that $0, u$ and $\tau=S$ satisfy the assumptions of the Lemma 3.1 if we choose $t=S+\delta$. Let, therefore $G$ be the set of radii given by the Lemma. We want, for $i$ sufficiently large, choose a $\beta$ such that the following conditions hold:

(a) $\tau_{i}(1-\beta)|a|=\tau_{i}(1-\beta)(1+\kappa)^{-1}|v|$ belongs to $G$, so that there exists a trajectory as in Lemma 3.1;

(b) The restriction on $\partial Q_{i, \beta}$ of the function $u_{t}$ is a Lipschitz function $\zeta$;

(c) $u_{t}$ is approximately differentiable at $\mathcal{H}^{1}$-a.e. point $x \in \partial Q_{i, \beta}$, and satisfies $H\left(x, \nabla u_{t}(x)\right) \leq 0$

(d) The derivative of $\zeta$ corresponds, $\mathcal{H}^{1}$-a.e. on $x \in \partial Q_{i, \beta}$, to the tangential component of $\nabla u_{t}$.

According to Proposition 2.1, the last three conditions are satisfied for a.e. $\beta$ such that $\partial Q_{i, \beta} \cap \gamma(t)=\emptyset$. Since

$$
\lim _{r \downarrow 0} \frac{\mathcal{H}^{1}\left(B_{r}(0) \cap \gamma(t)\right)}{r}=0
$$

and

$$
\lim _{r \downarrow 0} \frac{\mathcal{L}^{1}(G \cap[0, r])}{r}=0
$$


the existence of such a $\beta$ is guaranteed if $i$ is sufficiently large.

Now, we choose such a $\beta=\beta(i)$ for every $i$ and set $Q^{i}:=Q_{i, \beta(i)}$. Note that $y_{i}\left(s_{i}\right) \in Q^{i}$ if $i$ is large enough. Moreover, since $y_{i}(0)=x(0)$ and $x(0) \neq 0$, we have $y_{i}(0) \notin Q^{i}$, for any $i$ large enough. Thus, for large $i$ 's, there is a $\tilde{s}_{i}<s_{i}$ such that $y_{i}\left(\tilde{s}_{i}\right) \in \partial Q^{i}$. Now we let $z:\left[S-\tau_{i}(1-\beta)(1+\kappa)^{-1}, S\right] \rightarrow \mathbb{R}^{2}$ be the trajectory given by the last assertion of Lemma 3.1, which is joining the points $z\left(S-\tau_{i}(1-\beta)(1+\kappa)^{-1}\right)=x(S)-\tau_{i}(1-\beta)(1,0)$ and $0=x(S)$. Note that the first point belongs to $\partial Q^{i}$.

Next, observe that the perimeter of $Q^{i}$ can be bounded by $10 \tau_{i} \beta$. If $\alpha$ is chosen sufficiently small, the number

$$
\omega:=S-\tau_{i}(1-\beta)(1+\kappa)^{-1}-\tilde{s}_{i}
$$

is larger than $5 \beta \tau_{i} / \lambda$. Indeed, we have the inequalities

$$
\begin{gathered}
5 \beta \tau_{i} \lambda^{-1} \leq 5 \alpha \tau_{i} \lambda^{-1} \\
\omega \geq S-\tau_{i}(1-\alpha)(1+\kappa)^{-1}-s_{i}=\tau_{i}\left[1-(1-\alpha)(1+\kappa)^{-1}\right] .
\end{gathered}
$$

Hence the inequality $\omega \geq 5 \beta \tau_{i} \lambda^{-1}$ holds whenever

$$
\frac{\kappa+\alpha}{1+\kappa} \geq \frac{5 \alpha}{\lambda}
$$

Thus, the choice of $\alpha$ depends only on $\kappa$ and $\lambda$, which were fixed a priori.

Having chosen $\alpha$ accordingly small, we can find a trajectory

$$
\varphi:\left[\tilde{s}_{i}, S-\tau_{i}(1-\beta)(1+\kappa)^{-1}\right] \quad \rightarrow \quad \partial Q^{i}
$$

which joins $\varphi\left(\tilde{s}_{i}\right)=y_{i}\left(\tilde{s}_{i}\right)$ and

$$
\varphi\left(S-\tau_{i}(1-\beta)(1+\kappa)^{-1}\right)=z\left(S-\tau_{i}(1-\beta)(1+\kappa)^{-1}\right)
$$

and satisfies $\dot{\varphi}(\sigma) \in F(\varphi(\sigma))$ for every $\sigma$.

We join $z$ and $\varphi$ into a single trajectory $z$ on $\left[\tilde{s}_{i}, S\right]$, for which we have the following conclusions:

- $w=u_{t} \circ z$ is Lipschitz;

- for a.e. $\sigma$, either $\dot{z}(\sigma)=0$ or $u_{t}$ is approximately differentiable at $z(\sigma)$ and the approximate differential satisfies $H\left(z(\sigma), \nabla u_{t}(z(\sigma))\right) \leq 0$;

- for a.e. $\sigma$, either $\dot{w}(\sigma)=0$ or $\frac{d}{d \sigma} u_{t} \circ z(\sigma)=\nabla u_{t}(z(\sigma)) \cdot \dot{z}(\sigma)$ for a.e..

Next join the trajectory $\left.y_{i}\right|_{\left[0, \tilde{s}_{i}\right]}$ to the trajectory $z$ in order to build a new trajectory $y$. We claim that $y$ satisfies the requirements (P1)-(P3), thus showing that $S \in \mathcal{P}$. Indeed, $y$ satisfies all the requirements with $u_{t}=u_{S+\delta}$ in place of $u_{T+\delta}$. Thus, the computations (42) and (43) are still valid if we replace $T$ with $S$ and we infer $u_{S+\delta}(y(\sigma)) \leq \sigma \leq S<S+\delta$ for every $\sigma$. Therefore, the properties (P1)-(P3) with the desired value $T \geq S$ can be easily inferred from the following facts, which are easy consequences of the definitions of approximate differentiability and approximate continuity. Assume $a \in \mathbb{R}$ and $u_{a}(x)<a$. Then

- If $u_{a}$ is approximately continuous at $x$, so is any $u_{b}$ with $b>a$;

- If $u_{a}$ is approximately differentiable at $x$, so is any $u_{b}$ with $b>a$ and the corresponding approximate differentials coincide. 
This completes the proof that $S \in \mathcal{P}$.

\section{Step 2. The maximal element of $\mathcal{P}$ is $T$.}

Let $S$ be the maximal element. Then, it is obvious that $x(s) \neq x(S)$ for every $s>S$. In particular, if $S<T$, we must have $x(T) \neq x(S)$. Assume by contradiction that $S<T$ and, for $s>S$, consider the vectors

$$
v(s):=\frac{x(s)-x(S)}{s-S} .
$$

Recall that $B_{2 \delta}(\dot{x}(\sigma)) \in F(x(\sigma))$. By our assumptions on the multifunction $F$, it follows easily that $B_{\delta}(x(s)) \subset F(x(S))$ provided $s$ is sufficiently close to $S$. Therefore, we can apply Lemma 3.1. Given the set of radii $G$, it follows that, for any $\varepsilon>0$, there is $0<s<S+\varepsilon$ with $|s-S||v(s)| \in G$. We can therefore construct a zig-zag curve $z:[S, s] \rightarrow \mathbb{R}^{2}$ satisfying the assumptions of the Lemma with $t=S+\delta$, with $z(S)=x(S)$ and $z(s)=z(S)+(s-S) v(s)=x(s)$. Now, since $S \in \mathcal{P}$, there is a trajectory $y:[0, S] \rightarrow \mathbb{R}^{2}$ satisfying (P1), (P2) and (P3) with $y(S)=x(S)$. On the other hand, joining $z$ and $y$ into one single trajectory $\tilde{y}$, we can argue as in the previous step to conclude that $\tilde{y}:[0, s] \rightarrow \mathbb{R}^{2}$ satisfies (P1), (P2) and (P3). Since $\tilde{y}(s)=x(s)$, this implies that $s \in \mathcal{P}$, thus contradicting the maximality of $S$.

\section{Proof of Corollary 1.6}

Let $\left\{\gamma^{k}\right\}$ be a minimizing sequence of admissible strategies for the functional $J$. Consider the completions $\eta^{k}$ of $\gamma^{k}$. Then, $R_{\infty}^{\gamma^{k}} \supset R_{\infty}^{\eta^{k}}$ (because, by Theorem $1.5 T^{\gamma^{k}} \leq T^{\eta^{k}}$ ). Moreover, $\mathcal{H}^{1}\left(\eta_{\infty}^{k} \backslash \gamma_{\infty}^{k}\right)=0$. Thus, we conclude $J\left(\gamma^{k}\right) \geq J\left(\eta^{k}\right)$. Therefore, without loss of generality we can assume that the minimizing sequence of strategies $\left\{\gamma^{k}\right\}$ consists of complete strategies.

Consider the corresponding maximum time functions $T^{k}:=T^{\gamma^{k}}$. Note that the functions $T^{k}$ belong to the space of functions $G S B V$ (see Section 4.5 of [1]; this space is just a variant of the space of SBV functions introduced by Ambrosio and De Giorgi). Note also that $\left|D T_{t}^{k}\right| \leq \lambda^{-1} \mathcal{L}^{2}+t \mathcal{H}^{1}\llcorner\gamma(t)$. This uniform bound allows to apply the compactness theorem for GSBV functions (see Theorem 4.36 of [1]), which is just a variant of the SBV compactness Theorem of Ambrosio and De Giorgi. Hence, after passing to a subsequence, we can assume that $T^{k}$ converges pointwise a.e. to a function $u$ satisfying the following properties:

(a) $u_{t}$ is an SBV function for every $t$;

(b) $J_{u_{t}}$ is a rectifiable set and

$$
\int_{J_{u_{t}}} \psi d \mathcal{H}^{1} \leq \liminf _{k} \int_{J_{T_{t}^{k}}} \psi d \mathcal{H}^{1} \leq t
$$

(see Theorem 5.22 of [1]);

(c) $\nabla T_{t}^{k}$ converges weakly, in every $L^{p}$ with $p<\infty$, to $\nabla u_{t}$ (see Corollary 5.31 of [1]). For each $t$, denote by $\gamma(t)$ the set of points where the precise representative of $u_{t}$ is not approximately continuous. It is not difficult to see that $\gamma(t) \subset \gamma(s)$ for every $s>t$. 
Moreover, by Proposition 2.7, $\mathcal{H}^{1}\left(\gamma(t) \backslash J_{u_{t}}\right)=0$. It follows, therefore, from (b) that $\gamma(t)$ satisfies (H2) and, hence, it is an admissible strategy.

Note next that, $H$ is a continuous function and that $H(x, \cdot)$ is convex for every $x$. Then, the property $H\left(x, \nabla T_{t}^{k}(x)\right) \leq 0$ for a.e. $x$ implies, by (c), $H\left(x, \nabla u_{t}(x)\right) \leq 0$ for a.e. $x$. Thus, $u \in \mathcal{S}^{\gamma}$. So, if we consider the completion $\gamma^{c}$ of $\gamma$, we conclude $T^{\gamma^{c}} \geq u$.

Since $T^{k}$ converges pointwise a.e. to $u$, we conclude that

$$
\mathbf{1}_{\{u<\infty\}}(x) \leq \liminf _{k \uparrow \infty} \mathbf{1}_{\left\{T^{k}<\infty\right\}}(x) \quad \text { for a.e. } x .
$$

Thus, recall that $\alpha \geq 0$ and use Fatou's Lemma to conclude

$$
\begin{aligned}
\int_{R_{\infty}^{\gamma^{c}}} \alpha d \mathcal{L}^{2} & =\int_{\left\{T^{\gamma^{c}}<\infty\right\}} \alpha d \mathcal{L}^{2} \leq \int_{\{u<\infty\}} \alpha d \mathcal{L}^{2} \\
& \leq \liminf _{k \uparrow \infty} \int_{\left\{T^{k}<\infty\right\}} \alpha d \mathcal{L}^{2}=\liminf _{k \uparrow \infty} \int_{R_{\infty}^{\gamma^{k}}} \alpha d \mathcal{L}^{2} .
\end{aligned}
$$

On the other hand, by the Semicontinuity Theorem for SBV functions (see again Theorem 5.22 of [1]),

$$
\int_{J_{u_{t}}} \beta d \mathcal{H}^{1} \leq \liminf _{k \uparrow \infty} \int_{J_{T_{t}^{k}}} \beta d \mathcal{H}^{1} \leq \liminf _{k \uparrow \infty} \int_{\gamma_{\infty}^{k}} \beta d \mathcal{H}^{1}
$$

Since

we conclude that

$$
\int_{\gamma_{\infty}^{c}} \beta d \mathcal{H}^{1}=\sup _{t<\infty} \int_{J_{u_{t}}} \beta d \mathcal{H}^{1}
$$

$$
\int_{\gamma_{\infty}^{c}} \beta d \mathcal{H}^{1} \leq \liminf _{k \uparrow \infty} \int_{\gamma_{\infty}^{k}} \beta d \mathcal{H}^{1}
$$

From (44) and (45) it follows trivially that $J\left(\gamma^{c}\right) \leq \liminf _{k} J\left(\gamma^{k}\right)$. Hence, $\gamma^{c}$ is the desired minimizer.

\section{REFERENCES}

[1] L.Ambrosio, N.Fusco \& D.PAllara: Functions of bounded variation and free discontinuity problems. Oxford Mathematical Monographs, 2000.

[2] J.P.Aubin \& A.Cellina: Differential Inclusions., Springer-Verlag, Berlin, 1984.

[3] M.BARdi \& I.CAPuzzo-DolcetTA: Optimal control and viscosity solutions of Hamilton-JacobiBellman equations., Birkhuser, Boston, 1997.

[4] M.Bardi, M.G.Crandall, L.C.Evans, H.M.Soner \& E.Souganidis: Viscosity Solutions and Applications, Lecture Notes in Mathematics Vol. 1660, Springer-Verlag, Berlin, Heidelberg, 1997.

[5] A.Bressan: Differential inclusions and the control of forest fires. J. Differential Equations 243 (2007), no. 2, pp.179-207.

[6] A.Bressan, M.Burago, A.Friend \& J.Jou: Blocking strategies for a fire control problem. Anal. Appl. (Singap.) 6 (2008), no. 3, pp.229-246.

[7] A.Bressan \& C.De Lellis: Existence of Optimal Strategies for a Fire Confinement Problem, Comm. Pure Appl. Math. 62 (2009), no. 6, pp.789-830.

[8] A.Bressan \& T.WANG: Equivalent formulation and numerical analysis of fire confinement problem., Preprint, 2008. 
[9] A.Bressan \& T.Wang: The minimum speed for a blocking problem on a half plane., J. Math. Anal. Appl. 356 (2009), no. 1, pp.133-144.

[10] L.C.Evans: Partial differential equations. Graduate Studies in Mathemathics 319, AMS, 1991.

[11] L.C.Evans \& P.E.Souganidis: Differential games and representation formulas for solutions of Hamilton-Jacobi-Isaacs equations. Indiana Univ. Math. J. 33 (1984), pp. 773-797.

[12] H.Federer: Geometric Measure Theory. Die Grundlehren der mathematischen Wissenschaften, Band 153. Springer-Verlag, New York, 1969.

ZURICH UNIVERSITY

E-mail address: delellis@math.unizh.ch and roger.robyr@math.uzh.ch 